\title{
ATENDIMENTOS EM UM AMBULATÓRIO DE SAÚDE POR CAUSAS SENSÍVEIS À ATENÇÃO BÁSICA
}

\section{PRIMARY HEALTH CARE RELATED CAUSES IN AMBULATORY CARE}

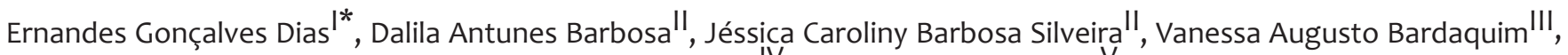 \\ Lyliane Martins Campos ${ }^{\mathrm{IV}}$, Maiza Barbosa CaldeiraV
}

Resumo. A Atenção Básica é a porta de entrada preferencial para os serviços de saúde e tem a capacidade de coordenar o cuidado dos usuários sob responsabilidade das Estratégias Saúde da Família. Os Ambulatórios são caracterizados como postos integrantes dos serviços de urgências e devem estar preparados para atender as demandas que necessitam de avaliação rápida e estabilização do quadro agudo. Objetivou-se investigar as demandas de atendimentos de saúde sensíveis à Atenção Básica apresentadas por usuários de um Ambulatório da cidade de Mato Verde, Minas Gerais, Brasil. Trata-se de um estudo descritivo e exploratório de natureza qualitativa. Os dados foram coletados entre agosto e setembro de 2019, com 15 usuários submetidos a entrevista semiestruturada e analisados mediante Análise do Conteúdo. Os resultados mostraram que os participantes tinham entre 20 e 63 anos de idade, a maioria eram casados, pardos, possuíam ensino médio completo e renda mensal bruta de $\mathrm{R} \$ 998,00$. Relataram procurar a Unidade Básica de Saúde quando apresentaram algum sinal ou sintoma e para a renovação de receitas médicas. Procuraram atendimento no Ambulatório devido à necessidade de agendamento prévio para atendimento na Atenção Básica e em virtude da manifestação de sintomas associados a problemas de saúde que parecem não se caracterizarem como atendimentos de urgência ou emergência. Conclui-se que a migração de usuários para atendimento no Ambulatório sem que sua demanda caracterize situação de urgência ou emergência representa um problema que parece cultural nessa população e também na gestão. Tais achados indicam que este estudo possa oferecer subsídios aos profissionais de saúde, bem como aos gestores na implementação de estratégias para corrigir lacunas assistenciais, sensibilizar a população a procurar o nível de atendimento adequado à sua necessidade, para melhorar o acesso e a resolutividade dos serviços de saúde.

PALAVRAS-CHAVE: Assistência Ambulatorial. Instituições de Assistência Ambulatorial. Atenção Primária à Saúde. Nível de Saúde.

Abstract. Primary Care is the preferred gateway to health services and is able to coordinate patient care under the Family Health Strategies responsibility. Ambulatories are integrated facilities to the emergency services and must be prepared to meet the demands that require rapid assessment and stabilization of acute conditions. The objective was to investigate the health care demands relevant to primary care brought up by patients of an ambulatory facility in the city of Mato Verde, Minas Gerais, Brazil. This is a qualitative, descriptive, and exploratory study. Data were collected between August and September 2019, with 15 users submitted to semi-structured interviews and analyzed using Content Analysis. The results showed that the participants were between 20 and 63 years old, married, mixed-race, high school educated, and which gross monthly income was the minimum wage (998.00 reais at the time). They reported to seek the Primary Care Unit when they showed any signs or symptoms and for medical prescriptions updating. They sought care at the Ambulatory due to the need for prior scheduling at the Primary Care Unit and due to the outbreak of health problem associated symptoms that did not seem to indicate urgent or emergency care necessity. It is concluded that patient migration seeking care at the Ambulatory without urgent or emergent demand represents an issue that seems to be cultural in this population and in the health care management. Such findings suggest that this study may contribute to health professionals, as well as managers in implementing strategies to fill gaps in care, to educate the population to seek the appropriate care to their needs, to improve access and effectiveness of health services.

KEYWORDS: Ambulatory Care. Ambulatory Care Facilities. Primary Health Care. Health Status.

IEnfermeiro, Mestre em Ciências. Faculdade Verde Norte (Favenorte). Departamento de Enfermagem. CEP: 39527-000, Mato Verde, Minas Gerais, Brasil. *Autor correspondente: ernandesgdias@yahoo.com.br. ORCID ID: 0000-0003-4126-9383.

IIGraduanda em Enfermagem, Faculdade Verde Norte (Favenorte). Departamento de Enfermagem. CEP: 39527-000, Mato Verde, Minas Gerais, Brasil . ORCID ID: 0000-0001-6292-1970; 0000-0002-7773-3021.

III Enfermeira, Doutora em Ciências. Universidade de São Paulo (USP). Departamento de Enfermagem. CEP: 14040-902, Ribeirão Preto, São Paulo, Brasi ORCID ID: 0000-0003-2179-552X.

IV Enfermeira, Especialista em Saúde Coletiva. Faculdade Verde Norte (Favenorte). Departamento de Enfermagem. CEP: 39527-000, Mato Verde Minas Gerais, Brasil. ORCID ID: 0000-0002-9476-2377.

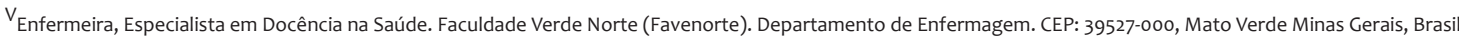
ORCID ID: 0000-0001-5444-6372 


\section{INTRODUÇÃO}

A Atenção Básica ( $A B)$ se constrói a partir de práticas de saúde individuais e coletivas que abrangem a promoção, prevenção, proteção, diagnóstico, tratamento, reabilitação, redução de danos, cuidados paliativos e vigilância em saúde. São elaboradas mediante ações de cuidado integrado e gestão qualificada, exercida por uma equipe multiprofissional destinada à população em território definido. ${ }^{1}$ No Brasil, tem-se vigente a Estratégia Saúde da Família (ESF) como modelo para reestruturar os níveis assistenciais e melhorar o acesso e a qualidade da $A B .^{2}$

$A A B$ é a principal porta de acesso da população às Redes de Atenção à Saúde(RAS), é coordenadora do cuidado e ordenadora das ações e serviços disponibilizados. $\mathrm{Na}$ perspectiva do Sistema Único de Saúde (SUS) e da RAS, a AB deve operacionalizar os princípios da universalidade, equidade e integralidade, bem como, as diretrizes da regionalização e hierarquização, territorialização, população adscrita, cuidado centrado na pessoa, resolutividade, ordenação da rede, longitudinalidade e coordenação do cuidado e participação da comunidade. $^{1}$

Nesta perspectiva, faz-se necessário esforços para fortalecer o atendimento na $A B$ a fim de melhorar o seu funcionamento e para dar direcionamento ao cuidado. O fortalecimento da $A B$ se consolida através de incentivos aos profissionais e esclarecimento aos usuários sobre qual serviço procurar no momento de manifestação de suas necessidades. ${ }^{3}$ Isto porque, muitas vezes, a alta demanda de usuários nos serviços de Urgência/Emergência (UE) é caracterizada por atendimentos de problemas de saúde que poderiam ser resolvidos na $A B .4$
O reconhecimento da relevância da organização das RAS a partir da $A B$ impulsionou $O$ desenvolvimento de propostas de avaliação de sua efetividade para a redução da morbimortalidade por diferentes condições. Nos anos 1990, foi proposto o termo "condições sensíveis" à $A B$ para designar um conjunto de problemas de saúde, para os quais a ação nesse nível de atenção diminuiria o risco de internações, por meio de prevenção de doenças, diagnóstico e tratamento precoce de condições agudas, controle e acompanhamento de condições crônica. ${ }^{5}$

As condições sensíveis à $A B$ refletem um conjunto de circunstâncias de saúde para as quais um cuidado efetivo, em momento oportuno, reduz desfechos desfavoráveis, como as internações hospitalares. Dentre os indicadores disponíveis para observação de desempenho da $A B$ tem-se o indicador Internações por Condições Sensíveis à Atenção Primária (ICSAP) o qual constitui uma medida indireta da efetividade da $A B$ e sua organização. ${ }^{6}$

A expansão da ESF causou impacto positivo na redução de internações por condições sensíveis à $A B$ em municípios brasileiros. No entanto, os índices de internações por essas condições no Brasil permanecem elevados, acima de $20 \%$ das internações, em comparação a outros países com sistemas de saúde universais, tais como Espanha e Austrália, que apresentam taxas em torno de $7 \%$ a $13 \%$, respectivamente. ${ }^{5}$

Frente a essas considerações, este estudo caracteriza-se como oportunidade de investigação indireta do acesso aos serviços da $A B$ e os motivos pelos quais os usuários buscam o serviço do Ambulatório para atendimento, sendo o resultado um 
importante indicador de resolutividade da $A B$ no município de Mato Verde, Minas Gerais (MG), Brasil. Assim, o estudo teve como objetivo investigar as demandas de

\section{MATERIAL E MÉTODOS}

Trata-se de um estudo descritivo e exploratório, de natureza qualitativa realizado com usuários do Ambulatório Municipal de Mato Verde-MG, que tiveram atendimento de demanda sensível à $A B$, residentes em Mato Verde, com idade entre 20 e 63 anos e que tinham funções cognitivas preservadas para responder à entrevista. Foram excluídos da pesquisa, os usuários que após triados preenchiam critérios para atendimento no Ambulatório, como as urgências médicas.

O município de Mato Verde está situado no norte de MG, possui 12.684 habitantes e densidade demográfica de 26,86 hab. $/ \mathrm{km}^{2}$. A AB do município está organizada em seis ESF, sendo quatro urbanas. As equipes são compostas por médico clínico geral, enfermeira, cirurgião dentista, auxiliar de saúde bucal, técnico de enfermagem, gerente administrativo e agentes comunitários de saúde que cobrem $100 \%$ do território urbano e rural do município.

O Ambulatório tem uma equipe composta por plantonistas médicos, enfermeiros, técnicos de enfermagem, técnico em radiologia entre outros, funciona 24 horas por dia, sete dias por semana e atende à demanda de forma espontânea ou referenciada pela ESF. Na cidade em estudo não existe hospital, assim o Ambulatório atende demandas de menor complexidade, mas não compatíveis com a capacidade de atendimento da $A B$ local.

O método de seleção do usuário foi aleatório simples e convencional de acordo com a sua disponibilidade e interesse no atendimentos de saúde sensíveis à $A B$, apresentadas por usuários do Ambulatório Municipal da cidade de Mato Verde. momento da coleta dos dados. Foram selecionados, através da ficha da triagem, preenchida pelo enfermeiro, quando o paciente buscou o Ambulatório. Através da ficha de triagem, identificou-se os usuários atendidos no estabelecimento e que poderiam ser assistidos pela $A B$ por não apresentar quadro clínico que caracterizasse condição de atendimento no Ambulatório.

Os dados foram coletados entre agosto e setembro de 2019, a partir de um roteiro de entrevista semiestruturada, elaborado pelos pesquisadores com duas questões disparadoras, aplicado individualmente aos usuários que atenderam aos critérios de seleção do estudo, até que se obteve saturação nos discursos.

$O$ roteiro de entrevista teve como questões disparadoras: por quais motivos você buscou atendimento no Pronto Atendimento? Como é sua relação (acesso e vínculo) com a equipe da $A B$ ?

As entrevistas foram realizadas na sala de triagem do Ambulatório em dias e horários alternados, no período matutino e vespertino, devido coincidir com o horário de funcionamento das Unidades de Saúde da $A B$ e tiveram duração média de 15 minutos. Foram gravadas em áudio, por meio do uso de um aplicativo de voz e, posteriormente, foram transcritas para organização e análise dos dados.

Os dados foram organizados, categorizados e analisados mediante "Análise do Conteúdo" na perspectiva de Bardin. ${ }^{7}$ 
A análise perpassou a compreensão e familiarização com os dados transcritos; busca e revisão de temas (assuntos gerais presentes nas falas dos usuários); definição e nomeação dos núcleos de registro e seleção dos fragmentos para interpretação e discussão dos dados.

Para resguardar a identidade dos entrevistados, os nomes destes foram substituídos por pseudônimos acompanhados de suas respectivas idades, na apresentação do conteúdo. O conteúdo do material empírico resultou em duas categorias de análise: "Acesso à Unidade de Saúde" e "A

\section{RESULTADOS E DISCUSSÃO}

\section{Caracterização dos participantes}

Participaram do estudo 15 usuários, 14 destes, do sexo feminino. A idade variou entre 20 e 63 anos, a maioria era casada $(n=9)$, pardas $(n=8)$, com ensino médio completo $(n=8)$ e renda mensal bruta de um saláriomínimo, $\mathrm{R} \$$ 998,00 ( $\mathrm{n}=8)$. Os participantes eram oriundos das quatro ESF da zona urbana da cidade.

\section{Acesso à Unidade de Saúde}

Os usuários relataram que procuram as Unidades de Saúde da $A B$ quando apresentam algum sinal ou sintoma específico e para renovar receitas. Esse comportamento remete à ideia de uma cultura curativista, o que pode ser uma dificuldade para realizar um trabalho preventivo, conforme preconizado para a $A B$.

"Só quando eu estou doente. Eu só vou quando eu tô muito doente, não vou lá toda hora". (Jasmim, 45 anos) procura pelo ambulatório e as preferências do usuário".

Todos os procedimentos metodológicos obedeceram aos padrões estabelecidos pela Resolução 466/2012 do Conselho Nacional de Saúde. O projeto de pesquisa foi aprovado pelo Comitê de Ética em Pesquisa da Universidade Estadual de Montes Claros-MG, Brasil, pelo Parecer Consubstanciado N. 3.456.244, CAAE: 16404019.9.0000.5146. Os participantes assinaram um Termo de Consentimento Livre e Esclarecido para autorizar o uso dos dados coletados pelo estudo.
"Pra renovar receitas ou levar minha menina pra consultar". (Antúrio, 28 anos)

"Pra eu ir no postinho é difícil, só se tiver que levar a receita pro médico fazer de novo ou sentir alguma coisinha, porque tenho pressão alta". (Cravo, 60 anos)

Tais achados corroboram com os resultados de um estudo realizado em Unidades de ESF de um município catarinense, no qual identificaram que a maioria dos usuários procuravam o acolhimento em razão de uma sintomatologia. Os principais motivos foram consultas, exames e medicamentos. ${ }^{8}$

Quando o usuário procura os serviços de saúde já com a doença instalada, há indícios de um modelo de atendimento hospitalocêntrico, o qual tem se mostrado enraizado na população e se opõe à lógica assistencial da $A B$. Entende-se que tal atitude fragiliza as atividades promotoras de saúde, bem como de prevenção, contribui para perpetuar a representação social da 
função da $A B$ como um serviço de pronto -atendimento ou ambulatorial. ${ }^{9}$ É consenso que a $A B$ é a porta de entrada preferencial ao sistema de saúde. Para isso, faz-se necessário que o cidadão entenda que não deve procurála apenas quando estiver debilitado, isso porque a medicina praticada na $A B$ é de caráter preventivo. ${ }^{1}$

$\mathrm{Na}$ fala dos participantes, percebese confiança nos profissionais da Unidade de Saúde com quem realizam suas consultas, o que pode indicar que há vínculo estabelecido na relação profissional-paciente:

"[...] confio, principalmente as enfermeiras informa gente, eu confio nelas, na competência". (Margarida, 47 anos)

"[...] gosto muito do médico porque tem muito tempo que ele trabalha na unidade, muito tempo que ele é médico, então ele é muito bom". (Jasmim, 45 anos)

"Eu gosto dos profissionais lá do posto, são todos eles muitos bons no serviço. A gente tem amizade até demais [...]". (Cravina, 52 anos)

A atuação da ESF de forma territorializada promove o vínculo com a população por meio do fortalecimento das ações intersetoriais com o estímulo a participação da comunidade. A família torna-se o primeiro objeto da atenção, compreendida a partir do ambiente onde vive e de sua interação com o meio, bem como, das suas relações com os serviços de saúde onde está inserida, considerando-se o conjunto de determinantes sociais, políticos e econômicos que permeiam a atuação dos profissionais e os serviços de saúde. ${ }^{11}$

O vínculo se constitui num elo entre profissional-usuário e garante segurança ao atendimento de suas necessidades, de tal forma que o usuário acredita e confia no profissional. Essa relação requer, de ambos, compartilhamento de momentos de conversação, escuta, acolhimento, trocas e responsabilização em torno dos problemas que o afligem. ${ }^{12}$

O tempo de atuação do profissional médico na ESF remete a ideia da existência de vínculo com os usuários desse serviço de saúde. Nesse sentido, a permanência dos profissionais de saúde na equipe, em especial de médicos e enfermeiros, é considerada um dos fatores críticos para o sucesso da ESF. A rotatividade desses profissionais pode comprometer a efetividade do modelo e prejudicar a qualidade da assistência e a satisfação dos usuários. ${ }^{13}$

\section{A procura pelo ambulatório e as preferências do usuário}

Os participantes afirmaram procurar atendimento no ambulatório devido à necessidade de agendamento prévio para atendimento na ESF e em virtude da manifestação de sintomas como febre, tosse, cefaleia, dor na garganta, associados a problemas de saúde. No entanto, os motivos da procura parecem não se caracterizarem como atendimentos de UE.

"Uma vez eu me lembro, foi quando eu tava com uma tosse muito forte há três dias, aí eu procurei ambulatório". (Jasmim, 45 anos)

"Porque eu fui na Unidade de Saúde e tinha que agendar e aí eu tive que ir no ambulatório. Em casos de tosse, febre e dores de cabeça". (Girassol, 39 anos)

"Às vezes eu venho pra cá quando tenho tosse, já vim também com dor de garganta. Agora mesmo tô sentindo a garganta doer". (Gardênia, 32 anos) 
As principais queixas de usuários atendidos na emergência de um hospital na região Norte do estado do Rio Grande do Sul (BR) era caracterizada por demandas de baixa complexidade, como hipertermia, lombalgia, dores abdominais e de garganta. ${ }^{14}$ Em um Hospital Geral de Pernambuco (BR) foi constatada a mesma situação, ou seja, a maioria dos atendimentos poderiam ser realizados na $A B$ por não se caracterizar como urgências. Os usuários utilizavam os serviços hospitalares de UE por entenderem que esta alternativa é mais resolutiva, pois recebem um somatório de recursos, como consultas, remédios, exames, procedimentos de enfermagem e internações. ${ }^{15}$

A necessidade de agendamento parece indicar falha na organização de um processo de trabalho mais efetivo na $A B$, dá-se a impressão de que não há atendimento para a demanda espontânea. Em estudo realizado em um município de médio porte do Estado do Rio Grande do Sul (BR) percebeu-se que os principais motivos da escolha de procurar o serviço de urgência eram relacionados a uma experiência negativa ao procurar as ESF, que esteve relacionada ao agendamento para atendimento, a falta de fichas e horários de funcionamento restrito. ${ }^{16}$

Aparece nos relatos dos entrevistados que o atendimento no ambulatório é mais rápido, não há necessidade de agendamento e ainda, certeza da presença do profissional médico. Merece destaque a observação de que a falta de médico na ESF acarreta procura pelo serviço de UE por motivos pelos quais não condizem com o nível de atenção e interfere na percepção de resolutividade entre os níveis de serviços. Tal fato, observa-se nas falas dos entrevistados:

"Porque na UBS tem que marcar a consulta e no ambulatório vocêéatendida mais rápido". (Amarílis, 20 anos)
"[...] a gente chega lá e eles não deixa a gente sair sem atender, sempre tem médico e no postinho nem todos os dias tem médico". (Begônia, 63 anos)

"Aqui não precisa agendar consulta e ficar um tempão esperando pra me atender e toda vez tem o médico aqui pra nós consultar". (Lírio, 45 anos)

Em um estudo realizado com usuários de um pronto atendimento, concluíram que os principais motivos referidos ao serviço, pelos usuários, foram a demora no atendimento e ausência do médico na ESF. Tal situação encontrada aponta fragilidades nos serviços da $A B$, pois esta deveria ser a porta de entrada preferencial para o sistema de saúde. ${ }^{17}$

Usuários de serviços de UE em Joinville, Santa Catarina (BR), indicaram como justificativas para tal, a indisponibilidade de vagas nas agendas de consultas médicas na ESF, falta de medicações para alívio imediato da dor, demora no agendamento e a coincidência de turnos de trabalho dos usuários com os horários de funcionamento da rede básica de saúde. ${ }^{18}$ Nesse sentido, o horário de atendimento acessível, exames realizados com agilidade, atendimento rápido, instalações agradáveis e equipadas, enfim, atendimento efetivo e resolutivo são motivos de preferência do usuário pelo UE. ${ }^{19}$

Resultados de um estudo, realizado em um hospital de Curitiba, Paraná (BR) corrobora com esta pesquisa ao indicar que $O$ principal motivo pelos quais os pacientes não urgentes se dirigem à UE em detrimento da $A B$ é por considerarem o atendimento mais resolutivo e mais rápido. ${ }^{20}$ Nesse tocante, é imperativo discutir a resolubilidade da $A B$, uma vez que parecem não conseguir atender aos usuários de forma adequada, o que acarreta procura, pelos usuários, aos serviços de UE de forma inadequada. 
A resolutividade é a capacidade de o serviço promover uma solução para os problemas dos usuários de forma adequada, no local mais próximo de sua residência ou encaminhando-os para onde suas necessidades possam ser atendidas conforme o nível de complexidade. Os profissionais de saúde das ESF devem garantir a resolutividade dos problemas dos usuários a partir da corresponsabilização pela atenção às necessidades de saúde e acesso contínuo aos demais níveis de assistência, quando necessário. ${ }^{21}$

\section{CONCLUSÃO}

A migração de usuários para atendimento no Pronto Atendimento, sem que sua demanda caracterize situação de Urgência e Emergência, representa um problema que parece cultural nesta população e também na gestão, já que perpassam problemas de organização de processo de trabalho na Atenção Básica e conscientização da população sobre as responsabilidades e competências de cada nível de atenção.

O estudo tem como limitações o fato de ter sido realizado em uma cidade de pequeno porte com uma demanda relativamente pequena para os serviços do Ambulatório. Porém, traz contribuições importantes para esta área do conhecimento,

\section{REFERÊNCIAS BIBLIOGRÁFICAS}

1. Brasil. Ministério da Saúde. Portaria ${ }^{\circ}$ 2.436. Aprova a Política Nacional de Atenção Básica, estabelecendo a revisão de diretrizes para a organização da Atenção Básica, no âmbito do Sistema Único de Saúde (SUS). Brasília, 21 set. 2017.

2. Costa JSD, Pattussi MP, Morimoto T, Arruda JS,
A $A B$ tem caráter coordenador e atribuição de resolução, organização e responsabilização pelo cuidado, além do dever de ser resolutiva. ${ }^{1}$ Os Hospitais e as UE devem ser utilizados apenas quando exijam maior complexidade tecnológica. Porém, é percebido que a hierarquia estabelecida não é obedecida pelos usuários, os quais continuam uma busca preferencial pelos serviços de UE o que preocupa os gestores de saúde porque acarretam uma sobrecarga de trabalho desses serviços e compromete a qualidade da assistência nos casos realmente urgentes. ${ }^{22}$

além de trazer à tona novas inquietações a serem respondidas em estudos futuros, a saber; buscar estimar o quanto a capacidade de atendimento do Ambulatório é ocupada para atender demandas da Atenção Básica.

Este estudo pode oferecer subsídios aos profissionais de saúde da Atenção Básica, em especial aos enfermeiros que acabam absorvendo a maior demanda de atendimentos da população, bem como, aos gestores para implementar estratégias que busquem corrigir as lacunas assistenciais e sensibilizar a população a procurar o nível de atendimento adequado a sua necessidade para melhorar o acesso e a resolutividade dos serviços de saúde.

Bratkowski GR, Sopelsa M. et al. Tendência das internações por condição sensível à atenção primária e fatores associados em Porto Alegre, RS, Brasil. Ciênc Saúde Coletiva. 2016;21(4):1289-96.

3. Oliveira GS. Superlotação das urgências eestratégias de gestão de crise: uma revisão de literatura. 
Cad grad. 2017;4(2):115-26.

4. Altino RC. Uso da rede de urgência e emergência e suas conexões com as unidades de atenção básica: uma análise transversal. 68f. Tese (doutorado). Universidade Estadual Paulista "Júlio de Mesquita Filho", Faculdade de Medicina de Botucatu. Botucatu, 2017.

5. Castro ALB, Andrade CLT, Machado CV, Lima LD. Condições socioeconômicas, oferta de médicos e internações por condições sensíveis à atenção primária em grandes municípios do Brasil. Cad Saúde Pública. 2015;31(11):2353-66.

6. Pinto ECP, Sirtoli R, Silva LL, Menolli PVS. A estratégia de saúde da família e as internações por condições sensíveis à atenção primária no Paraná: série temporal, 2007-2016. Rev Saúde Pública. 2018;1(2):35-47.

7. Bardin L. Análise de conteúdo. $4^{\mathrm{a}}$ ed. Lisboa: Edições 70, 2011.

8. Felchilcher E, Araújo G, Traverso MED. Perfil dos usuários de uma unidade básica de saúde do meiooeste catarinense. Unoesc Ciênc. 2015;6(2):223-30.

9. Brito GEG, Mendes ACG, SANTOS NETO PM. O objeto de trabalho na Estratégia Saúde da Família. Interface, Botucatu, 2018;22(64):77-86.

10. Silveira ALP. Unidade Básica de Saúde (UBS). Rede HumanizaSus [online]. set., 2016.

11. Santos RCA, Miranda FAN. Importância do vínculo entre profissional-usuário na estratégia de saúde da família. Rev Enferm UFSM. 2016;6(3):350-59.

12. Ilha S, Dias MV, Backes DS, Backes MTS. Vínculo profissional-usuário em uma equipe da Estratégia Saúde da Família. Ciênc Cuid Saúde. 2014;3(1):556-62.

13. Tonelli BQ, Leal APR, Tonelli WFQ, Veloso DCMD, Gonçalves DP, Tonelli SQ. Rotatividade de profissionais da Estratégia Saúde da Família no município de Montes Claros, Minas Gerais, Brasil.
Rev Fac Odontol UPF. 2018;23(2):180-85.

14. Marco VR, Strapazzon M, Silva LAA, Lorenzoni AMC, Oliveski CC, Silva LES. Necessidades clínicas de atendimento evidenciadas em uma unidade de emergência: um olhar sob ótica do sistema de avaliação de risco. XXIV Seminário de Iniciação Científica. Salão do Conhecimento, Unijuí. 2016.

15. Camerro A, Alves EC, Camerro NMMS, Nogueira LDP. Perfil do atendimento de serviços de urgência e emergência. Rev Fafibe On-Line. 2015;8(1):515-24.

16. Fonseca DF, Silva SO, Lima SBS, Fonseca GGP, Carvalho JL, Fonseca HF. Acessibilidade organizacional da atenção primária: a saúde como determinante da demanda em um pronto socorro. In: Anais do Terceiro Seminário Internacional Tecendo Redes na Enfermagem e na Saúde. 2019. Santa Maria.

17. Pícoli RP, Cazola LHO, Maurer NMJS. Service users classified as risk level 'blue' in an emergency department. Cogitare Enferm. 2016;21(1):01-07.

18. Lopes GSSPL, Sardagna MC, IERVOLINO SA. Motivos que levam os homens a procurar um serviço de pronto atendimento. Rev Enferm. 2017;20(2):15165.

19. Freire $A B$, Fernandes DL, Moro JS, Kneipp MM, Cardoso CM, Lima SBS. Serviços de urgência e emergência: quais os motivos que levam o usuário aos pronto-atendimento? Saúde (Santa Maria). 2015;41(1):195-00.

20. Pimentel SK, Soares MC, Mazepa MM, Kato PVK, Lima RL, Soares MEC. Análise dos motivos para uso do serviço de urgência e emergência pelo paciente não grave. Rev Méd UFPR. 2016;3(3):109-13.

21. Farias DC, Celino SDM, Peixoto JBS, Barbosa ML, Costa GMC. Acolhimento e Resolubilidade das Urgências na Estratégia Saúde da Família. Rev bras educ. med. 2015;39(1):79-87.

22. Carvalho ACA, Morais ECLS. Análise das 
fragilidades da atenção básica enquanto porta de entrada para os serviços de saúde do SUS no Brasil. 31f. Trabalho de Conclusão de Curso. Graduação em Enfermagem. Universidade Tiradentes. Aracaju, 2015. 